\title{
Speed adaptation for a robot walking with a human
}

\author{
Emma Sviestins \\ Royal Institute of Technology \\ S-100 44 Stockholm \\ Sweden \\ emmasv@kth.se
}

\author{
Noriaki Mitsunaga \\ ATR IRC Laboratories \\ Kyoto 619-0288 \\ Japan \\ mitunaga@atr.jp
}

\author{
Takayuki Kanda \\ ATR IRC Laboratories \\ Kyoto $619-0288$ \\ Japan \\ kanda@atr.jp
}

\begin{abstract}
We have taken steps towards developing a method that enables an interactive humanoid robot to adapt its speed to a walking human that it is moving together with. This is difficult because the human is simultaneously adapting to the robot. From a case study in human-human walking interaction we established a hypothesis about how to read a human's speed preference based on a relationship between humans' walking speed and their relative position in the direction of walking. We conducted an experiment with a humanoid robot, Robovie-IV, to verify this hypothesis. For 11 out of 15 subjects the results were consistent with the hypothesis. All six subjects satisfied with one or more of the speeds that our algorithm estimated and four of them answered one of them as the best one if the algorithm was allowed to give three options; but since there were confusing data for the algorithm it had difficulty deciding the best speed among the three. In the paper, we will discuss the difficulties and possibilities that we learned from this preliminary trial.
\end{abstract}

\section{Categories and Subject Descriptors}

H.5.2 [Information Interfaces and Presentation]: User Interfaces-Interaction Styles; I.2.9 [Artificial Intelligence]: Robotics

\section{General Terms}

Design, Human Factors

\section{INTRODUCTION}

Over the past several years many humanoid robots that can make sophisticated human-like expressions have been developed. Several research works have demonstrated use of human-like body properties for natural human-robot interaction, such as facial expression [1], head orientation [11], and body position [16]. We believe that humanoid robots will be suitable for our research on "communication robots" that behave as peer-partners to support daily human activ- ities based on advanced interaction capabilities [8]. In addition to providing physical support, these robots will supply support through communication, for instance guiding the way to some destination.

Adaptation and learning is considered an important research direction in human-robot interaction. For instance, Breazeal et al. developed a robot that is capable of learning from a human collaborative partner [2]. Suga et al. proposed a possibility of tracking preferred repertory of interaction by direct feedback from a human using an evolutionary learning method [14]. Mitsunaga et al. demonstrated that an interactive robot is capable of adjusting distance and timing of the interaction to the interacting human by reading discomfort signals that are subconsciously emitted by the human [10].

In this paper, we focus on the situation where a communication robot is moving together with a walking human. There are many applications for a robot moving together with a human, such as a museum guide $[3,4,7]$ or an assistant [6]. Navigation of a robot in the presence of humans is often studied [13, 15]. Personal space and distance among humans and robots are considered important [5, 12]. However, not much previous research has been conducted on an adaptation mechanism for a robot during walking.

There are three parameters to consider for adaptation during walking: movement speed, parallel distance (the distance in parallel with the moving direction), and side distance (the distance vertical to the direction of moving). Of these, movement speed is most important since if the distance is wrong the human can adjust it by him-/herself but if the speed is wrong the human will have to put up with it in order to walk together with the robot. This is especially important if the robot is meant to act as a social partner in the daily life of people. However, just as two humans walking together adjust their speed to each other, speed adaptation in a human-robot situation can be mutual. That is to say, a robot trying to adapt its speed to a human must take into consideration that the human might simultaneously be adapting to the robot. This complicates the matter as it is not safe for the robot to merely try to keep the same speed as the human. For instance, if the robot starts out slowly the human might keep a slow pace so as not to leave the robot behind.

To overcome this problem it is necessary to find some way 


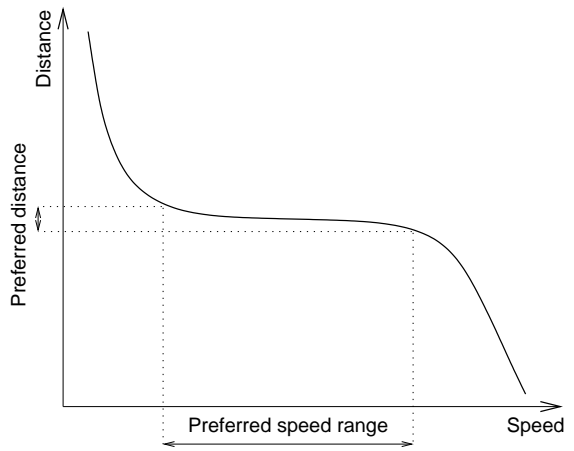

Figure 1: The relationship between walking speed and parallel distance according to our hypothesis.

that humans signal whether the speed they are currently keeping is comfortable for them or not. By studying humans walking together we have found a relationship between walking speed and relative position in the direction of walking that could give information about a person's true preference. We verify our idea by conducting an experiment with two humans walking together. Then we conduct a similar experiment with a human and a robot, and finally we try a simple speed estimation algorithm.

\section{ADAPTATION METHOD}

By studying people walking together we found that people seem to have a range of walking speeds which they are comfortable with. On the other hand, they appear to have a narrow range of what relative position in the walking direction to keep with respect to the person they are walking together with: some like to walk a little distance ahead or behind while others prefer to walk side by side. People seem to tend to adjust their speed in order to keep this position, unless the other person's speed is much too fast or too slow. This leads us to the idea that people will keep their preferred relative position as long as the walking speed is within their preferred range; when the speed is outside that range the relative position will change even though the person might still be keeping up with the other person. We sketch this proposed relationship in Fig. 1.

To adapt to a human's preference a robot first needs to gather some speed-position information changing its speed and observing the human's changes in position. It can then know whether or not its speed is in the preferred range. We propose an off-line preferred speed estimation method for adapting to a human's preference:

1. The robot tries several different movement speeds while walking with a human in order to gather speed-position information,

2. then it selects a speed from the interval within which the position does not change very much.

\section{HUMAN-HUMAN EXPERIMENT}

To confirm our ideas of how humans signal discomfort with the walking speed, we set up an experiment with two persons walking together, one of which was the experimenter.

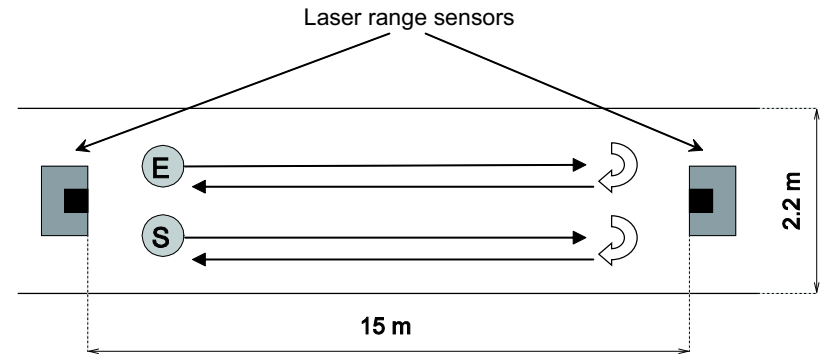

Figure 2: The experiment setup. 'E' and ' $S$ ' mark the experimenter and the subject respectively and the boxes represent the range sensors placed on tables. The experimenter and the subject moved back and forth in the measurement area.

\subsection{Experiment setup}

In this experiment the experimenter and a subject walked together back and forth along a stretch of corridor. (Fig. 2) Measurements of the persons' positions were taken using two SICK LMS-200 laser range sensors, placed approximately 15 meters apart at each end of the experiment area, at a height of $1.1 \mathrm{~m}$ above the floor. The data acquired while the persons were less than three meters from one of the ends of the experiment area was discarded to avoid taking position measurements while the persons were slowing down and turning around.

\subsection{Subjects and procedure}

The experiment was conducted with 12 subjects, 7 male and 5 female, with an average age of 21 years. We chose an intern at ATR as the experimenter. The subjects received instructions from an experiment assistant and were told that the experimenter was another subject. The purpose of this was to make the subjects feel that the person they were walking with was a person of equal status, since we believed that this would be the best condition for mutual interaction. The experiment consisted of two parts:

\section{Gathering speed-position data:}

The experimenter varied speed and distance as shown in Table 1; each parameter value was tested during one "lap": walking from one end to the other and back. The distance covered in one lap is about 20 meters excluding the areas for slowing down and turning. The subject was given two subsequent tasks to perform while walking. Both tasks consisted of solving arithmetic problems read by the experimenter; the tasks differed only in the level of difficulty of the problems. During each task the experimenter cycled through all parameter values in Table 1 once. The main purpose of the tasks was to provide a controlled form of interaction between experimenter and subject since we wanted to simulate a situation of talking while walking together. The purpose of having two tasks with different levels of difficulty was to find out if people's preference change when they are working on a difficult task. This is not treated in this paper, however.

2. Measuring preferred speed interval: To find out the subject's preferred speed interval, we 
Table 1: Approximate parameter values for the human-human experiment. Relative position denotes the relative position in the direction of walking of the subject to the experimenter. +100 means that the subject walked $100 \mathrm{~mm}$ ahead of the experimenter.

\begin{tabular}{ccc}
\hline $\begin{array}{c}\text { Lap no. } \\
\text { subj 1-6 }\end{array}$ & $\begin{array}{c}\text { Lap no. } \\
\text { subj 7-12 }\end{array}$ & $\begin{array}{c}\text { Rel. pos. } \\
{[\mathrm{mm}]}\end{array}$ \\
\hline 1 & 6 & 0 \\
2 & 7 & +100 \\
3 & 8 & +500 \\
4 & 9 & -100 \\
5 & 10 & -500 \\
\hline \hline Lap no. & Lap no. & Rel. pos. \\
subj 1-6 & subj 7-12 & [mm] \\
\hline 6 & 1 & 1000 \\
7 & 2 & 800 \\
8 & 3 & 600 \\
9 & 4 & 400 \\
10 & 5 & 300 \\
\hline
\end{tabular}

asked the subject to raise a blue or orange flag if the walking speed was too fast or too slow, respectively. The experimenter and the subject walked a total of four laps, two with the easy math task and two with the difficult one. The experimenter changed his walking speed in steps as above from $600 \mathrm{~mm} / \mathrm{s}$ to 1000 $\mathrm{mm} / \mathrm{s}$ in the first half of each lap, and from $600 \mathrm{~mm} / \mathrm{s}$ to $300 \mathrm{~mm} / \mathrm{s}$ in the second half.

\subsection{Results}

Due to technical problems we were only able to use data from 15 of the $24=(12 \times 2)$ experiments. 10 of the 15 speed-distance relation graphs give indications that our hypothesis is correct. Figs. 3 and 4 show such examples. In the figure, 'slow' and 'fast' lines indicate the average speed during the time span the subject held up the 'slow' and 'fast' flags respectively. For some subjects there are several 'slow' or 'fast' lines since subjects often raised flags at different speeds. As shown in Fig. 3, we consider subject 4, difficult task a successful case since the graph has the expected shape with plateau and negative slope. Fig. 4, subject 3 , difficult task also shows a graph that has roughly the right shape, though lacking a well-defined plateau and not being quite consistent with the subject's flagged speed preference.

Meanwhile, the other five graphs did not to fit with the hypothetic model. As shown in Fig. 5, subject 5, difficult task represents an unsuccessful case: the graph does not have the expected shape. For subject 11, easy task, the data points are scattered and no clear curve can be seen (Fig. 6). We believe that this is caused in part by low data quality but mainly by the difficulty for a human experimenter to keep a constant speed.

\section{HUMAN-ROBOT EXPERIMENT}

To test the results indicated by the human-human experiment in a human-robot situation we devised an experiment where the robot tries a range of speeds while the distance of the human to the robot is measured. We also tried out a simple offline speed estimation algorithm.

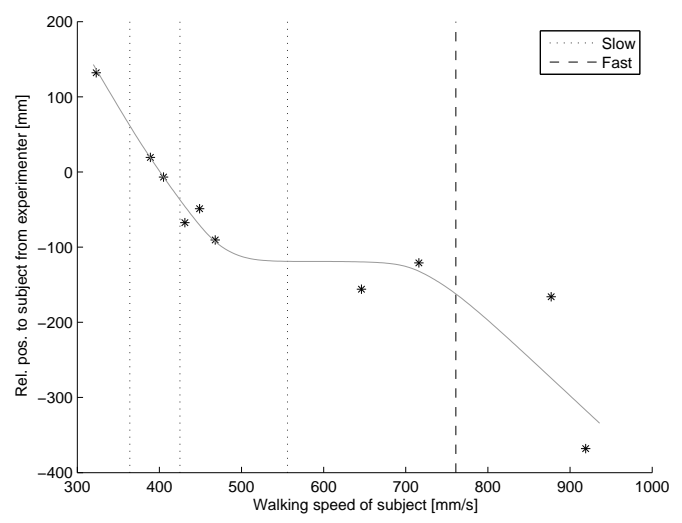

Figure 3: Case matched with the hypothesis: The speed-position relation and the preferred speed of subject 4 under hard math task. This graph supports our hypothesis.

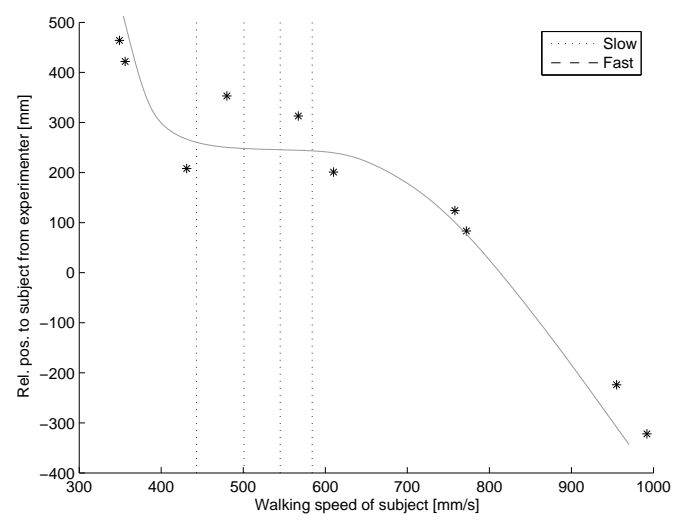

Figure 4: Case matched with the hypothesis: The speed-position relation and the preferred speed of subject 3 under hard math task.

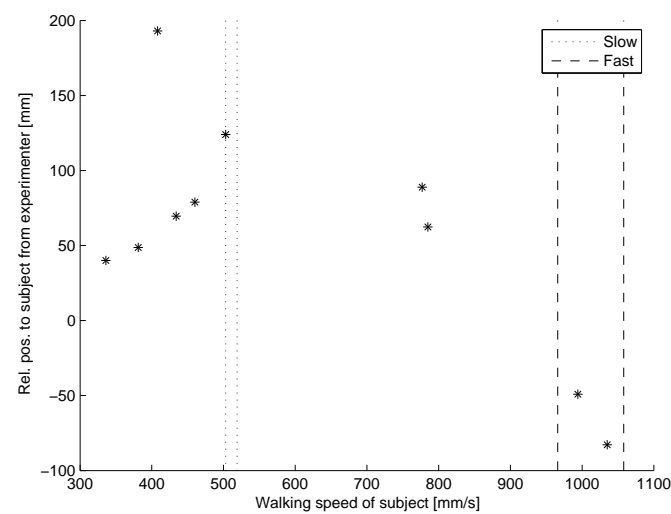

Figure 5: Case that did not match with the hypothesis: The speed-position relation and the preferred speed of subject 5 under hard math task. 


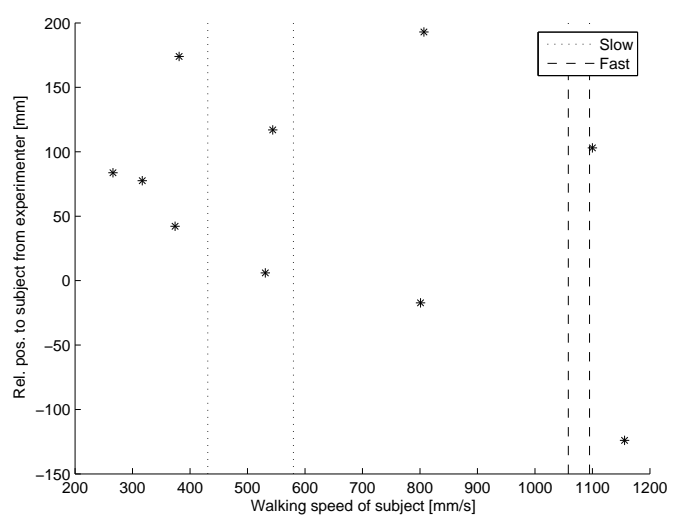

Figure 6: Case that positions varied too much: The speed-position relation and the preferred speed of subject 11 under easy math task.

\subsection{Estimation of preferred speed}

For the robot to be able to perform speed estimation it must first gather some data on the human's speed preference according to our speed-position relationship. In this experiment, Robovie-IV and the subject walked together while the robot gradually changed its speed.

The gathered speed-position data was then used to estimate the preferred speed. We used a simple algorithm which selected the speed interval with least negative distance/speed derivative. If there is a short interval with positive derivative at the lowest speeds the algorithm will exclude this range. The reason for this is that some subjects had shown a behavior of stopping and waiting for the robot when it was moving too slowly, which is outside of our hypothesis.

\subsection{Setup and robot platform}

The physical experiment setup was identical to the setup in the human-human experiment (Fig. 2) with two SICK LMS200 laser range sensors placed 15 meters apart in a corridor, except that the robot took the place of the experimenter.

The robot used throughout this research is the humanoid robot Robovie-IV [9] (Fig. 7), developed at ATR. It consists of a humanoid upper body on a differential drive platform capable of moving at $1 \mathrm{~m} / \mathrm{s}$. It is $1 \mathrm{~m}$ tall and is equipped with a variety of sensors such as cameras, microphones and skin sensors. However the only sensors used in this experiment were two Hokuyo laser range sensors mounted front and back on the base of the robot, which were used for obstacle detection, human tracking for the purpose of gaze meeting and localization by map matching. Robovie-IV is equipped with an on-board $\mathrm{PC}$ with a Pentium-M $2 \mathrm{GHz}$ CPU, running Linux. It has a speaker and text-to-speech software, enabling it to utter sentences in Japanese. Since the robot was too short to be registered by the range sensors it was fitted with a cylindrical paper hat as can be seen in Fig. 8.

\subsection{Procedure}

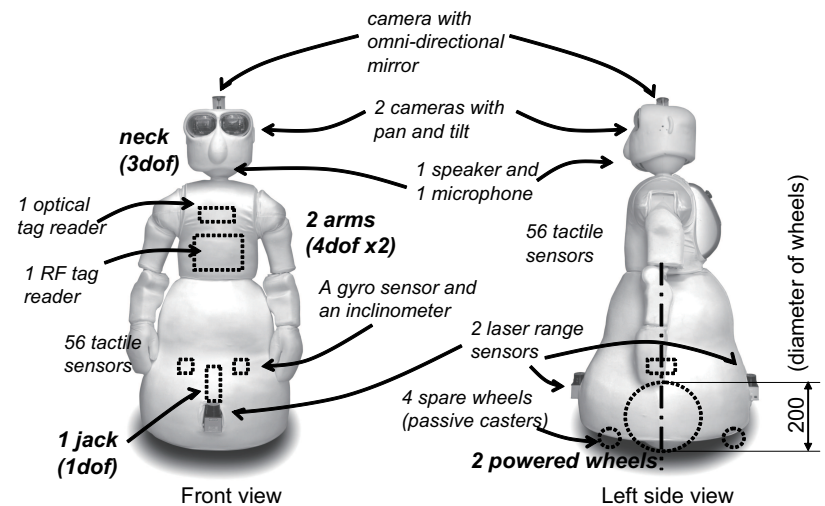

Figure 7: Overview of Robovie-IV, it has two powered wheels, two 4 d.o.f arms, a head, and sensors such as cameras, microphones, skin sensors, laser range sensors, and so on.

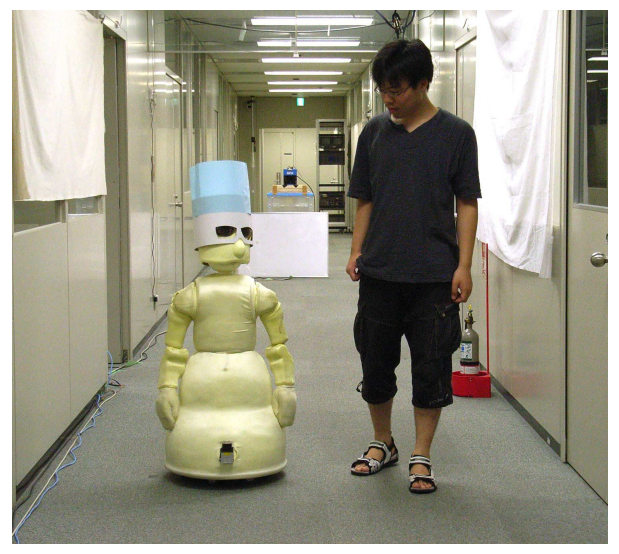

Figure 8: Robovie-IV, wearing his experiment hat, is walking along with a human

For people to perceive the robot as an interactive partner it should display some kind of interactive behavior. For this purpose, the robot gives the subject arithmetic problems as in the human-human experiment, to create a form of conversation. Additionally, the robot turns its head to look at the subject when it is speaking, as well as occasionally between utterances.

During the experiments the robot moved back and forth between the range sensors. At each end it would slow down, turn around and speed up again. During this time no measurements were taken.

The experiment was conducted at ATR using 15 subjects: 5 male and 10 female. The average age was 22 years. For the first 9 subjects, we verified that our hypothesis works in a human-robot situation with the following procedure:

\section{Gathering speed-position data:}

The robot cycled through speeds between $300 \mathrm{~mm} / \mathrm{s}$ and $1000 \mathrm{~mm} / \mathrm{s}$, with a step of $100 \mathrm{~mm} / \mathrm{s}$. It ran at each speed for 4 seconds: 1 second for changing speed and 3 seconds for taking measurements of the distance 
to the subject. If the end of the measurement area was reached before the 4 second interval was completed the robot would start over at that speed after turning around. The robot did a total of 10 laps.

2. Measuring preferred speed:

2a. Best speed: The robot ran each lap on a constant speed, one lap for each speed used in the human-human experiment (from $300 \mathrm{~mm} / \mathrm{s}$ to $1000 \mathrm{~mm} / \mathrm{s}$. See table 1). After each lap the subject was asked if this speed was comfortable for walking. Also we asked the subject which speed(s) was best after the all laps. If the subject answered more than one speed as the best we recorded all of them.

2b. Preferred speed interval: The robot cycled speeds as in "gathering speed-distance data" and the subject was asked to raise a blue or orange flag if the speed was too slow or too fast as in preferred speed interval measurement in humanhuman experiment.

For the last 6 subjects, we ran step 1 as above and fed the resulting speed-position data to the speed estimation algorithm. The output speeds of the algorithm were prepended to the list of speeds for step $2 \mathrm{a}$ and the experiment was continued as above.

\subsection{Speed-position data gathering results}

We plotted the experimental result of speed-position relations. An experimenter looked at the plotted figures and classified them into three categories: the shape with plateau (matched with the hypothetical model), the shape looked like a flat-negative-slope, and other shapes. Table 2 shows the classification result. We classified the results of steps 1 and 2 a. From the table, we see that the plot of steps 1 and $2 \mathrm{a}$ matched with the hypothesis for 11 and 9 subjects respectively. Figs. 9 through 12 show the speed-position relationships of steps 1 and $2 \mathrm{a}$ for subjects $8,16,15$, and 7 . The 'slow' and 'fast' lines indicate the speeds that the subject started to answer the speeds are slow and fast in step 2a respectively. The 'best' line indicates the speed that the subject selected as best one in step 2a. As in Fig. 9, subject 8's speed-position relation for step 1 and step 2a matched with the hypothesis. However, there are differences in the position where plateaus stay. Subject 16's relations (Fig. 10) are another example of a matched case. Here the plateau is shifted to the right and the negative slope is hidden since the subject 16 's preferred speed was $950[\mathrm{~mm} / \mathrm{s}]$. We classified the results of step 1 as "flat" and step 2a as "matched" for subject 15 (Fig. 11). Subject 15 preferred $600[\mathrm{~mm} / \mathrm{s}]$ and we see no plateau in step 1 . As for step $2 \mathrm{a}$, plateau seems to starting at between 600 and $800[\mathrm{~mm} / \mathrm{s}]$ which the robot did not try. We classified the results of step 1 as "matched" and step 2a as "flat" for subject 7 (Fig. 12). Subject 7 preferred $800[\mathrm{~mm} / \mathrm{s}]$ and we see slight plateau in step 1 . But the relationship changed to "flat" for step $2 \mathrm{a}$.

Overall, the speed-position graphs were closer to our theoretical graph in this experiment than in the human-human experiment. We believe that this is a result of better data

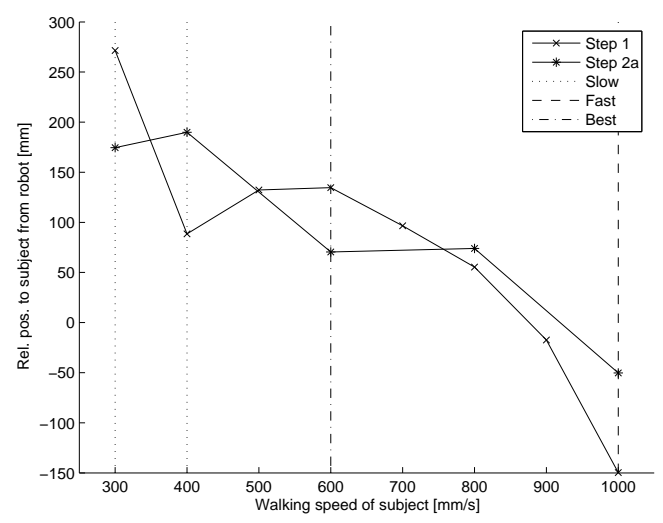

Figure 9: Case matched with the hypothesis: The speed-position relationship of subject 8 . Best indicates the speed that subject answered as the best. Slow/Fast indicates the starting speed that the subject claimed slow/fast.

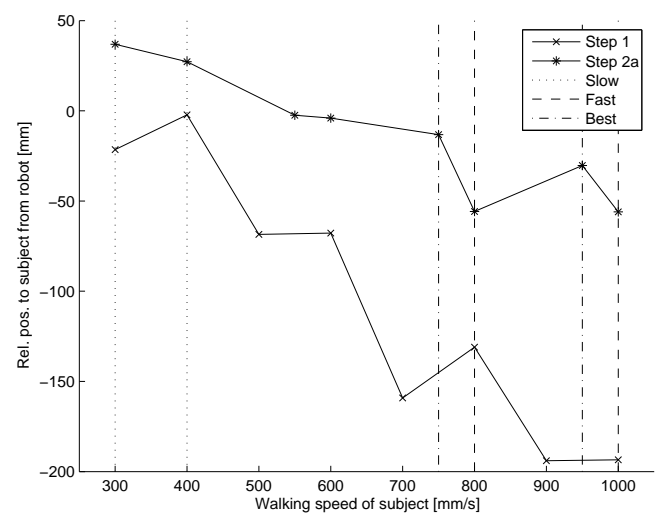

Figure 10: Case matched with the hypothesis: The speed-position relationship of subject 16 . The plateau can be seen in higher speed range and the negative slope is hidden.

quality: each data point in these graphs is an average of 2-3 measurements taken during 3 seconds each, whereas the in human-human plots each data point is a single measurement taken during half a lap. In general, we found the graphs from the experiment to be fairly consistent with our hypothesis. We note, however, that for seven subjects the graph has a plateau at the lowest speeds. We conclude that this comes from the subject being relatively far ahead of the robot and not wanting to leave him further behind at very slow speeds. Additionally, we note that two graphs have a positive slope at the highest speeds, which is contrary to our expectations.

\subsection{Speed estimation results}

The results of the speed estimation algorithm are shown in Table 3. The subject and the robot walked laps with speed(s) estimated by the algorithm (s1, s2, s3) and five fixed speeds (300 to $1000[\mathrm{~mm} / \mathrm{s}])$. In the table, the speed(s) that the subject answered as best are shown in bold type. 
Table 2: Classification result of the speed-position plots under two conditions; robot changed speed in a lap and robot kept constant speed in a lap

\begin{tabular}{c|c|c|c|c|c|c}
\multicolumn{2}{c|}{$\begin{array}{c}\text { Robot varied speed } \\
\text { (step 1 speed-position relation) }\end{array}$} & \multicolumn{2}{c|}{$\begin{array}{c}\text { Robot kept constant speed } \\
\text { (step 2a best speed measurement) }\end{array}$} & \\
\hline $\begin{array}{c}\text { with plateau } \\
\text { (matched with } \\
\text { the model) }\end{array}$ & $\begin{array}{c}\text { flat } \\
\text { negative } \\
\text { slope }\end{array}$ & other & $\begin{array}{c}\text { with plateau } \\
\text { (matched with } \\
\text { the model) }\end{array}$ & $\begin{array}{c}\text { flat } \\
\text { negative } \\
\text { slope }\end{array}$ & $\begin{array}{c}\text { other } \\
\text { other }\end{array}$ & $\begin{array}{c}\text { class } \\
\text { changed }\end{array}$ \\
\hline 11 & 4 & 0 & 9 & 5 & 1 & 8
\end{tabular}

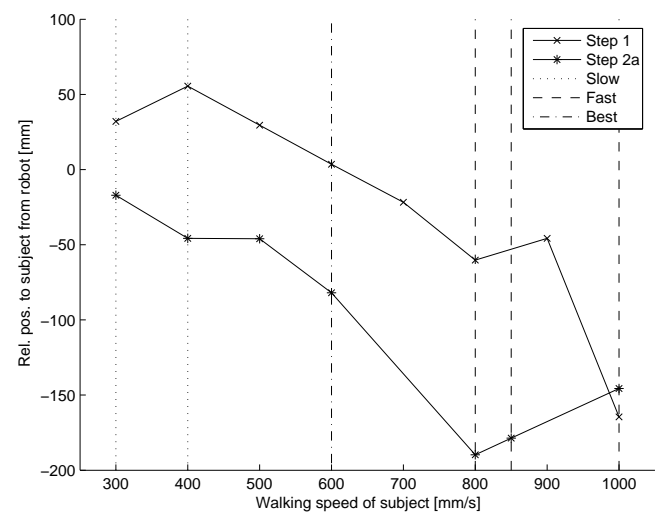

Figure 11: Case that step 1 is classified as "flat" and step 2a as "matched": The speed-position relationship of subject 15 .

Table 3: Result for the speed estimation algorithm in the human-robot experiment. Speeds marked as best by the subject are shown in bold type. All speeds are in $\mathrm{mm} / \mathrm{s}$.

\begin{tabular}{c|ccc|ccccc}
\hline Subj. & \multicolumn{3}{|c|}{ Alg. output } & \multicolumn{4}{c}{ Standard range } \\
& s1 & s2 & s3 & 300 & 400 & 600 & 800 & 1000 \\
\hline 13 & 550 & $\mathbf{9 0 0}$ & & & & & $\mathbf{8 0 0}$ & \\
14 & 650 & 850 & & & & $\mathbf{6 0 0}$ & & \\
15 & 500 & 850 & & & & $\mathbf{6 0 0}$ & & \\
16 & 550 & $\mathbf{7 5 0}$ & $\mathbf{9 5 0}$ & $\mathbf{3 0 0}$ & & & & \\
17 & 550 & $\mathbf{8 0 0}$ & & & & & $\mathbf{8 0 0}$ & \\
18 & $\mathbf{8 0 0}$ & & & & & & \\
\hline
\end{tabular}

We can see that four subjects chose one or more of the estimated speeds as the best one. The subjects 14 and 15 did not choose them as the best speed. However, since they said that the estimated speed of 650 and $500[\mathrm{~mm} / \mathrm{s}]$ are good speeds for them and their preferable speed $600[\mathrm{~mm} / \mathrm{s}]$ is close to the estimated speed, we can say that the algorithm also successfully estimated the preferred speed for these two subjects.

\section{DISCUSSIONS}

\subsection{How well does human-robot interaction data fit with the hypothetical model?}

It was revealed that for 11 out of 15 subjects the results were consistent with the hypothesis while for four subjects they were not. One explanation for this is that the choices of

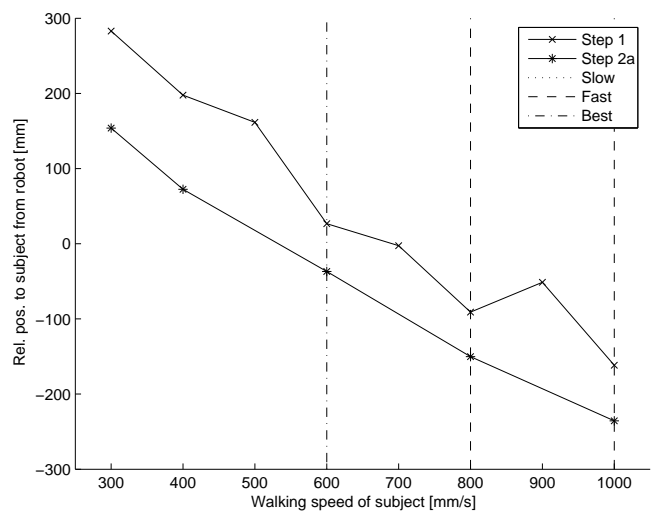

Figure 12: Case that step 1 is classified as "matched" and step $2 \mathrm{a}$ as "flat": The speed-position relationship of subject 7 .

speeds presented to the subjects were too sparse. Another is the relative position's dependency on how the robot changes its speeds. Contrary to our expectation, speed-position relationships differed in their values or shapes for most of the subjects as the Figs. 10,11, and 12 show. This indicates the importance of the "data gathering method" or how a robot changes its speed.

In addition, we found that the pattern was not consistent between constant-speed mode and variable-speed mode (Table. 2). When we measured the best speed, we used the constant-speed mode. As a result, eight subjects' speedposition pattern was changed from that of the variable-speed mode. At the variable speed, four subjects was categorized into the flat-negative-slope pattern, which means that we have a difficulty in estimating their preferable speed; at the constant speed mode, two of these four subjects categorized into the with plateau pattern. This implies that there is a possibility to accurately estimate people's preference if a robot appropriately changes its speed pattern between constant-speed and variable-speed.

Note that four of eleven subjects who were categorized in the with-plateau pattern at the variable speed moved into the flat-negative-slope pattern at the constant speed. Thus, it does not mean that the constant speed mode is better than the variable speed mode. In fact, the fitness of the model is better at the variable speed. This also suggests a robot to keep changing its speed to adjust its speed to a 


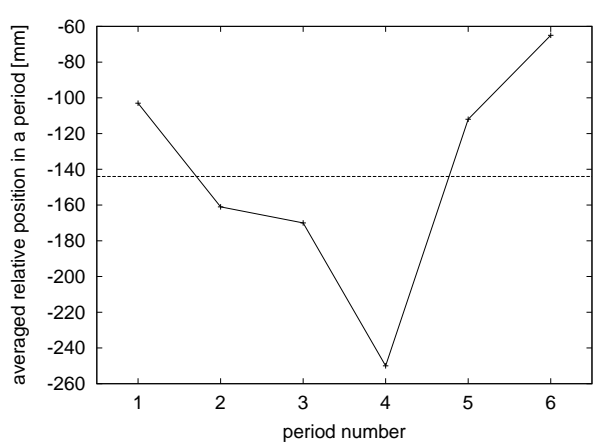

Figure 13: The changes of the averaged relative position in one lap (subject 18). Robovie moved in $600[\mathrm{~mm} / \mathrm{s}]$. The average is taken at every $4[\mathrm{~s}]$. This subject changed her relative position frequently. Averaged relative position in the lap was $-144[\mathrm{~mm}]$.

person as long as the reaction from the person fits with the with-plateau model. Probably, people are more conscious with the current speed when the speed is changed; and if the speed is constant for a while, they are accustomed to be at the speed due to humans' flexibility toward walking speed.

\subsection{Estimating subjects' preferred speed}

As shown in Table 3, our algorithm successfully predicted subjects' preferred speed if it was allowed to give three speeds. The subject who was presented three speeds preferred two of them. The three subjects who were presented two speeds preferred one of them and one subject preferred both. If we assume this study as a preliminary trial, this is a promising result. Since the range of preferable speed is narrow, the chance rate of making a successful guess is not very high. Thus, this is unlikely to happen by chance. On the other hand, the algorithm had difficulty deciding the best one among three speeds. As discussed in the previous section, the data did not fully fit the hypothetical model, which caused erroneous speeds. It is our future work to improve the algorithm so that it will provide the best speed with better precision.

\subsection{Oscillation of human behavior}

Furthermore, we analyzed the temporal aspect of the relative position and found that the distance sometimes oscillated. Figure 13 is a plot of relative positions of a subject and the robot during one lap (about $20 \mathrm{~m}$ ) of the experiment when the robot's speed was constant at $600 \mathrm{~mm} / \mathrm{s}$. The subject often changed her position, going in front of and behind the robot. On the contrary, Figure 14 shows the relative position of another subject where the robot's speed was also at $600 \mathrm{~mm} / \mathrm{s}$. This subject did not change her position so often and kept the distance relatively stably. This indicates that the robot may need to observe a person's reaction to its speed for a certain amount of time.

\subsection{Observations}

Through this study, we found that adaptation of a robot's speed to a human is even more difficult than we originally expected. Here we describe what we have learned that can give us insight for further studying such an adaptation method.

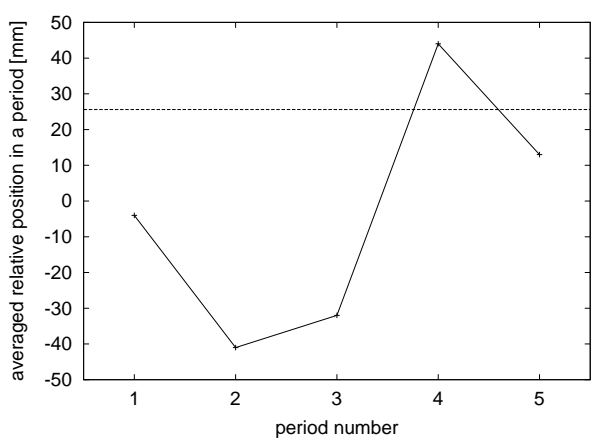

Figure 14: The changes of the averaged relative position in one lap (subject 16). Robovie moved in $600[\mathrm{~mm} / \mathrm{s}]$. The average is taken at every $4[\mathrm{~s}]$. This subject did not changed her relative position so much as the subject $18 \mathrm{did}$. Averaged relative position in the lap was $26[\mathrm{~mm}]$.

- People's preferred walking speed is not fixed, rather, it changes depending on task and situation. There is also a large individual variation. In our case where people are asked to perform a thinking task, some preferred very slow speed $(400 \mathrm{~mm} / \mathrm{s})$ while some preferred fast speed (more than $900 \mathrm{~mm} / \mathrm{s}$ ). Therefore it is motivated to make a robot that is capable of adjusting its speed to a person.

- Even though a person may at a glance seem to be satisfied with the speed of walking this is not necessarily the case. Although we carefully observed video recordings we could not find any obvious behavior such as facial expressions, gestures, rapid change of speed and distance, and so forth, that might suggest dissatisfaction with the walking speed. This indicates the need of an active method that tries a variety of speeds on a human and watches their behavior.

- Rather than being drawn from observations of obvious behavior, our hypothetical model about the relationship between speed and parallel distance is established from a case study. It fits with $11 / 15$ of human-robot interaction data.

- Observations of a person's relative position in the direction of walking gives us a better chance of predicting his/her preferred speed, although the fitness of the model was not so supportive. Note that it cannot find the best speed yet; further study will be required before we can use this technique for real applications.

- Observation of parallel distance gives us better chance of predicting his/her preferable speed. Note that it could not find the best speed yet. For $5 / 6$ subjects, it estimated two or three speeds. Thus, it requires further study to apply this technique for real applications.

- A comparison of the graphs from constant versus variable speed mode suggests that adjustment of speed step might be necessary. It seems that although people might be sensitive to speed changes they are insensitive to small differences between two speeds. 
- People's speed-position pattern changed between the variable-speed mode and the constant-speed mode. At the variable-speed mode, 11 out of 15 subjects fit the hypothetical model; but 4 of them shifted into the flatnegative-slope pattern at the constant speed. Regarding the rest of 4 subjects who were categorized in flatnegative-slope pattern at the variable-speed model, 2 subjects fit with the hypothetical model at the constantspeed mode. This suggests that we need to pay attention to the way how a robot indicates its speed.

- Some people often change his/her position within short movements of $20 \mathrm{~m}$ (about one lap in our experiment setting). This suggests that the robot needs to observe people's behavior for a certain amount of time.

\section{CONCLUSION}

We found a relationship between walking speed and relative position which we used to implement a speed estimation method that successfully found the preferred speed for all six subjects and the best speed for four of them even though the humans were adapting to the robot. However, not all subjects in our experiments displayed speed-position relationships consistent with our hypothesis. In future work it is important to investigate how far this method can be extended. It is also important to make the speed-position data gathering phase as short and smooth as possible, for example by not exploring more of the speed range than is necessary so as to avoid making the human feel discomfort.

\section{ACKNOWLEDGEMENTS}

This research was supported by the Ministry of Internal Affairs and Communications of Japan.

\section{ADDITIONAL AUTHORS}

Additional authors: Hiroshi Ishiguro (Graduate School of Engineering, Osaka University, email: ishiguro@ams . eng . osaka-u.ac. jp) and Norihiro Hagita (ATR IRC Laboratories, email: hagita@atr.jp).

\section{REFERENCES}

[1] Breazeal and B. Scassellati. A context-dependent attention system for a social robot. In Proc. Int. Joint Conf. on Artificial Intelligence, pages 1146-1151, 1999.

[2] C. Breazeal, D. Buchsbaum, J. Gray, D. Gatenby, and B. Blumberg. Learning from and about others: Towards using imitation to bootstrap the social understanding of others by robots. Artificial Life, 11(1-2), 2005

[3] W. Burgard, A. B. Cremers, D. Fox, D. Hähnel, G. Lakemeyer, D. Schulz, W. Steiner, , and S. Thrun. The interactive museum tour-guide robot. In Proc. National Conference on Artificial Intelligence (AAAI), 1998.

[4] R. S. et al. Robox at expo.02: A large scale installation of personal robots. Robotics and Autonomous Systems, pages 203-222, 2003.

[5] E. T. Hall. The Hidden Dimension. Anchor Books, 1990.
[6] H. Hüttenrauch, A. Green, M. Norman, and L. O. K. S. Eklund. Involving users in the design of a mobile office robot. IEEE Transactions on Systems, Man and Cybernetics, 34:113-124, 2004.

[7] T. Kanda, T. Hirano, D. Eaton, and H. Ishiguro. Interactive robots as social partners and peer tutors for children: A field trial. Human Computer Interaction, 19(1-2):61-84, 2004.

[8] T. Kanda, H. Ishiguro, M. Imai, , and T. Ono. Development and evaluation of interactive humanoid robots. In Proceedings of the IEEE, volume 92, pages 1839-1850, 2004.

[9] N. Mitsunaga, T. Miyashita, Y. Yoshikawa, H. Ishiguro, K. Kogure, and N. Hagita. Robovie-iv: A robot enhances co-experience. In Proceedings of the Workshop on Ubiquitous Experience Media at ISWC'05, pages 17-23, 2005.

[10] N. Mitsunaga, C. Smith, T. Kanda, H. Ishiguro, and N. Hagita. Robot behavior adaptation for human-robot interaction based on policy gradient reinforcement learning. In IEEE/RSJ International Conference on Intelligent Robots and Systems (IROS2005), pages 1594-1601, 2005.

[11] K. Nakadai, K. Hidai, H. Mizoguchi, H. G. Okuno, , and $\mathrm{H}$. Kitano. Real-time auditory and visual multiple-object tracking for robots. In International Joint Conference on Artificial Intelligence (IJCAI'01), pages 1425-1432, 2001.

[12] Y. Nakauchi and R. Simmons. A social robot that stands in line. Autonomous Robots, 12(3):313-324, 2002.

[13] E. A. Sisbot, R. Alami, T. Simeon, K. Dautenhahn, M. Walters, S. Woods, K. L. Koay, and C. Nehaniv. Navigation in the presence of humans. In IEEE International Conference on Humanoid Robots (Humanoids2005), pages 181-188, 2005.

[14] Y. Suga, Y. Ikuma, D. Nagao, T. Ogata, and S. Sugano. Evolution of human-robot communication in real world. In Proc. of IEEE/RSJ International Conference on Intelligent Robots and Systems (IROS 2005), pages 1482-1487, 2005.

[15] E. A. Topp and H. I. Christensen. Tracking for following and passing persons. In IEEE/RSJ International Conference on Intelligent Robots and Systems (IROS2005), pages 70-76, 2005.

[16] G. Trafton, A. Schultz, D. Perznowski, M. Bugajska, W. Adams, N. Cassimatis, and D. Brock. Children and robots learning to play hide and seek. In Prof. of the Conf. on Human-Robot Interaction (HRI2006), pages 242-249, 2006. 